\title{
Analysis of turbulent synthetic jet by dynamic mode decomposition
}

\author{
Tomáš Hyhlík ${ }^{1, \star}$, Hana Netřebská1, Jakub Devera ${ }^{1}$, and Radomír Kalinay ${ }^{1}$ \\ ${ }^{1}$ CTU in Prague, FME, Department of Fluid Dynamics and Thermodynamics, Technická 4, 16607 Prague
}

\begin{abstract}
The article deals with the analysis of CFD results of the turbulent synthetic jet. The numerical simulation of Large Eddy Simulation (LES) using commercial solver ANSYS CFX has been performed. The unsteady flow field is studied from the point of view of identification of the moving vortex ring, which has been identified both on the snapshots of flow field using swirling-strength criterion and using the Dynamic Mode Decomposition (DMD) of five periods. It is shown that travelling vortex ring vanishes due to interaction with vortex structures in the synthesised turbulent jet. DMD modes with multiple of the basic frequency of synthetic jet, which are connected with travelling vortex structure, have largest DMD amplitudes.
\end{abstract}

\section{Introduction}

This work can be understood as a continuation of previous works dealt with modal decomposition and analysis of synthetic jets, see e.g. works [1, 2].

The existence of synthetic jet is related to vortex ring creation. The created vortex ring is induced velocity in the direction outside from the orifice. The most commonly used parameters of the synthetic jet are based on so-called slug model, see e.g. [3, 4]. Velocity scale is defined as time averaged blowing orifice centerline velocity over an entire cycle

$$
U_{0}=\frac{1}{T} \int_{0}^{T_{E}} u_{0}(r=0, t) \mathrm{d} t,
$$

where $T_{E}$ is blowing time and $T=1 / f$ is oscillation period. Reynolds number can be determined using time averaged blowing orifice centerline velocity $U_{0}$ as

$$
R e=\frac{2 U_{0} D}{v}
$$

where $D$ is orifice diameter and $v$ is kinematic viscosity. Stokes number is defined as

$$
S=D \sqrt{\frac{2 \pi f}{v}}
$$

where $f$ is frequency.

The regime of synthetic jet with $R e=898$ and $S=$ 19.7 is choosen.

\section{Numerical Simulation}

Unsteady incompressible turbulent flow simulation has been performed using commercial solver ANSYS CFX.

\footnotetext{
^e-mail: tomas.hyhlik@fs.cvut.cz
}

The turbulence is simulated using LES on the computational mesh of about 740000 cells. The subgrid-scale viscosity is solved with Smagorinsky model with constant of the model 0.1. Central difference scheme is chosen as advection scheme. The temporal scheme is set as second order backward Euler. Both the flow in the orifice and actuator cavity are included in the simulation to get more accurate results. The effect of oscillating diaphragm is replaced by velocity boundary condition which should guarantee achievement of required Reynolds number

$$
u(t)=\frac{\pi}{2} \frac{\operatorname{Re} \eta}{\rho D} \frac{D^{2}}{D_{L}^{2}} \sin (2 \pi f t),
$$

where $\eta$ is dynamic viscosity, $\rho$ is air density and $D_{L}$ is inlet diameter i.e. diameter of oscillating diaphragm. The time step is set to $1 / 1000$ of the period. The simulation is run for 65 periods to get the developed flow field.

Results of the numerical simulation are shown in the figure 1 , where there are visualised vortex structures at five phases of the period after $1 / 5$ of the period. Vortex structures are visualised using swirling-strength criterion. It is possible to see the movement of the vortex ring which is going from left to right. The vortex ring created in the blowing part of the period is very stable structure because it is not possible to see its spontaneous brake up. There are vortex structures in the right part of the figure 1 connected with the turbulent jet which is created by travelling vortex ring. The tail like vortex structure is created behind travelling vortex ring. The travelling vortex ring seems to disappear due to interaction with vortex structures in the turbulent jet flow, as can be seen in the figure 1 .

\section{Dynamic Mode Decomposition}

DMD is a kind of modal decomposition method. Let the velocity field be represented by ensemble of snapshots sampling at interval $\Delta t$ with a form of matrix $\mathbf{V}_{1}^{N}=$ 


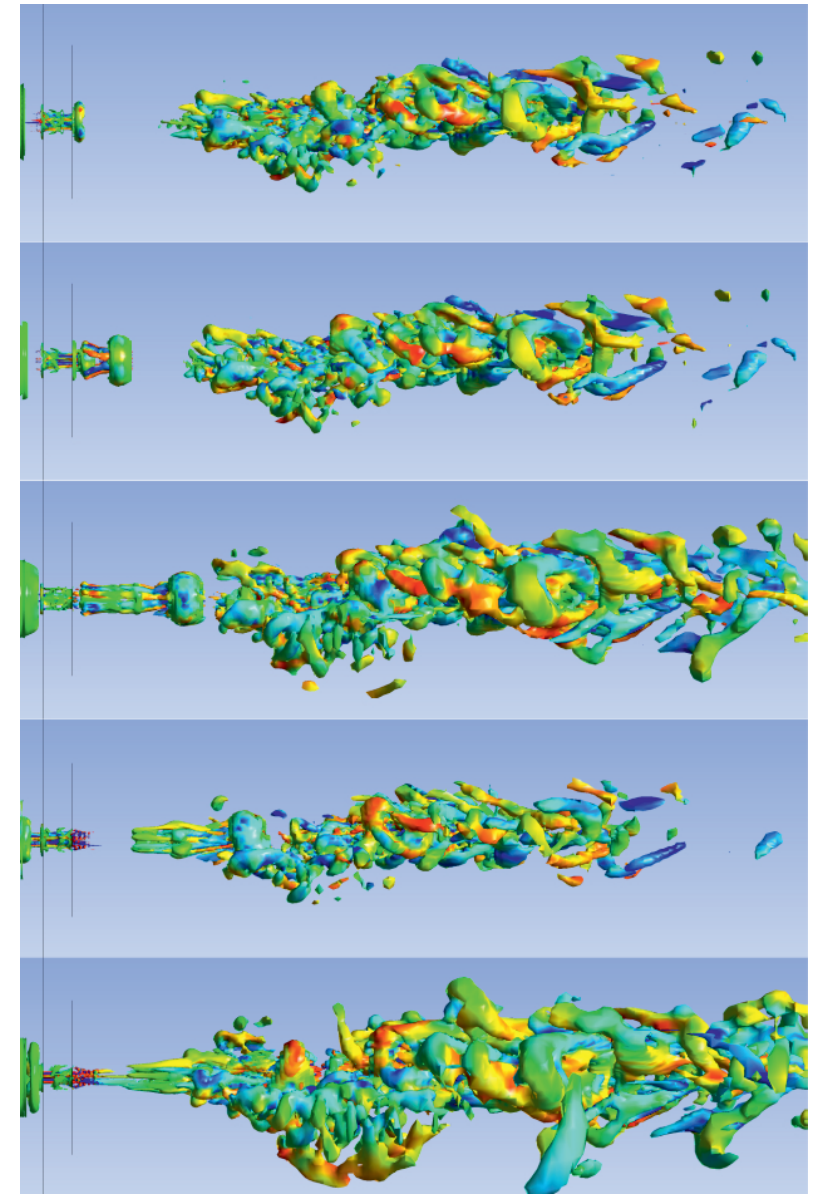

Figure 1. Vortex structures visualized using swirling-strength criterion; temporal step is $1 / 5$ of the period

$\left\{\mathbf{v}_{1}, \mathbf{v}_{2}, \ldots, \mathbf{v}_{N}\right\}$, where the column vector $\mathbf{v}_{j}$ contains velocity field at $j$ th time step. Let's consider linear mapping A that maps each snapshot to the next one in the following way

$$
\mathbf{v}_{j+1}=\mathbf{A} \mathbf{v}_{j} .
$$

This mapping is assumed to be same over the full sampling interval $[0,(N-1) \Delta t]$. The assumption of a constant mapping between the snapshots $\mathbf{v}_{j}$ allows us to formulate the sequence of flow fields as a Krylov sequence

$$
\mathbf{V}_{1}^{N}=\left\{\mathbf{v}_{1}, \mathbf{A} \mathbf{v}_{1}, \mathbf{A}^{2} \mathbf{v}_{1}, \ldots, \mathbf{A}^{N-1} \mathbf{v}_{1}\right\} .
$$

The goal of DMD is the extraction of dynamic characteristics such as eigenvalues and eigenvectors which are described by linear operator $\mathbf{A}$ based on sequence $\mathbf{V}_{1}^{N}$.

Robustness is achieved by preprocessing step using singular value decomposition of data sequence [5]. The algorithm is working with a similar matrix to matrix $\mathbf{A}$. Eigenvalues of similar matrix approximate eigenvalues of full mapping matrix $\mathbf{A}$ and provide temporal dynamics of the flow field.

\section{Results of DMD}

DMD decomposition of the flow field is based on unsteady data from last five periods. Only one hundred snapshots are taken from each period because of a large amount of the processed data. Figure 2 shows amplitudes of several DMD modes. Largest amplitude is connected with steady mode with zero frequency. There are a few DMD modes with low amplitude in figure 2, but largest amplitudes are connected with the basic frequency of synthetic jet $f=96 \mathrm{~Hz}$ and with its multiples. Figures 3 and 4 show the spatial structure of the DMD mode connected with synthetic jet frequency. Figures 5 and 6 show the spatial structure of the DMD mode connected with double of synthetic jet frequency. Figure 7 shows the spatial structure of the DMD mode connected with the triple of synthetic jet frequency. The real and imaginary part of the mentioned spatial DMD modes have similar scales. The spatial structures of real and imaginary parts are space shifted and are very similar, there are alternating local minimums, where the structures are finer for higher DMD mode frequency. The alternating local minimums like structure diminishes about fifteen diameters from the orifice. This kind of behaviour documents travelling vortex ring-like structure, where vortex ring diminishes about fifteen diameters from the orifice.

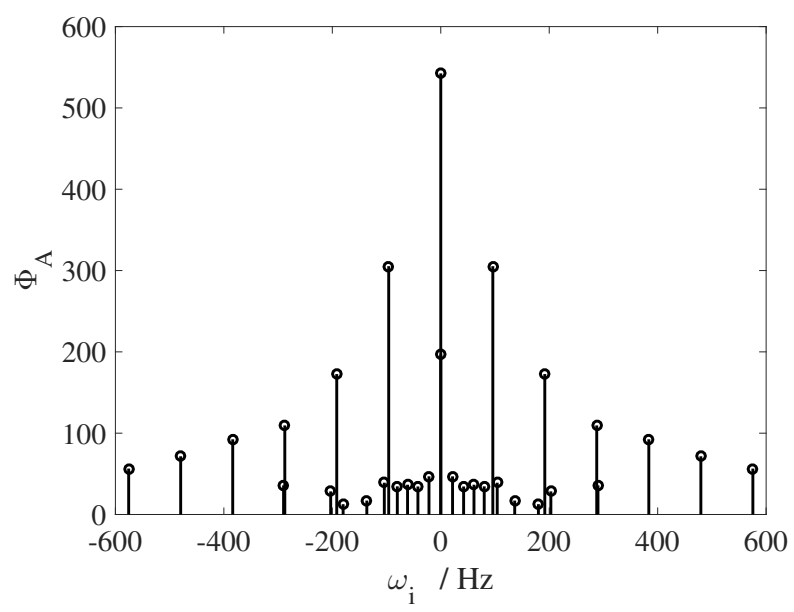

Figure 2. DMD amplitudes

\section{Conclusions}

The vortex structures in the turbulent synthetic jet are studied using swirling-strength criterion and using DMD decomposition method. DMD method is able to identify mainly the travelling vortex ring. The absence of DMD modes with a higher frequency which are connected with the turbulent jet is probably caused by low sampling frequency which is chosen with respect to the necessary amount of data. The results of vortex visualisation are based on the swirling-strength criterion, where the vortex structures calculated using LES are shown.

\section{Acknowledgement}

The support from Czech Science Foundation under Grant No. 14-08888S is gratefully acknowledged. 


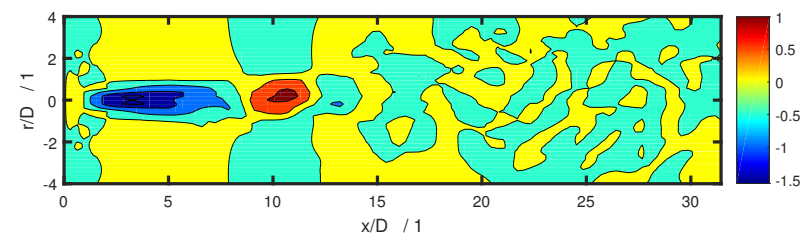

(a) xy plane, $x$ velocity, real part of DMD mode

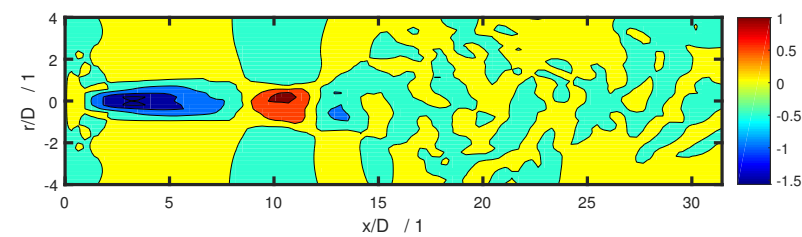

(c) xz plane, $x$ velocity, real part of DMD mode

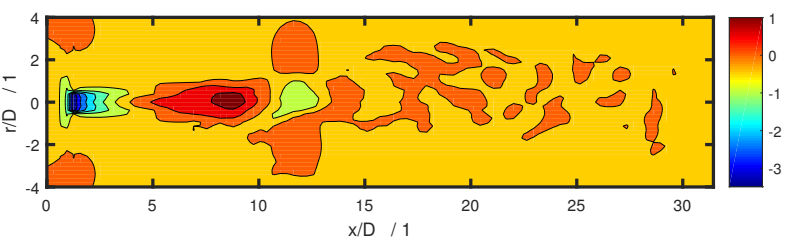

(b) xy plane, $x$ velocity, imaginary part of DMD mode

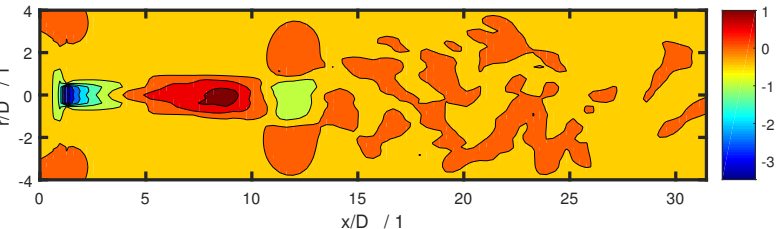

(d) $x z$ plane, $x$ velocity, imaginary part of DMD mode

Figure 3. Dynamic mode with $f=96 \mathrm{~Hz}$; axial velocity component $\mathrm{x}$

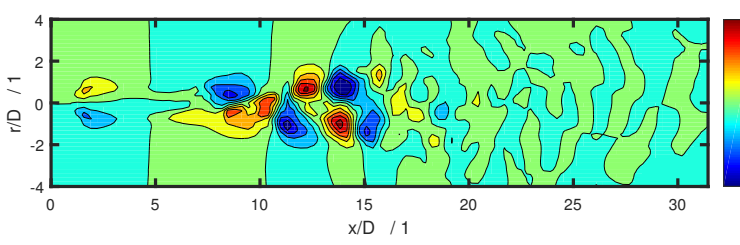

(a) xz plane, $z$ velocity, real part of DMD mode

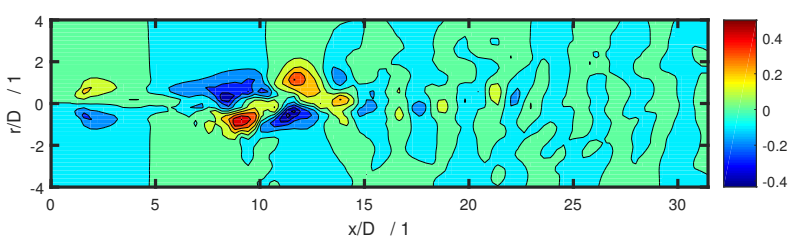

(c) xy plane, y velocity, real part of DMD mode

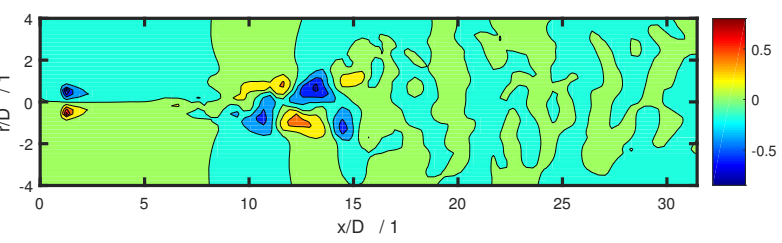

(b) xz plane, $\mathrm{z}$ velocity, imaginary part of DMD mode

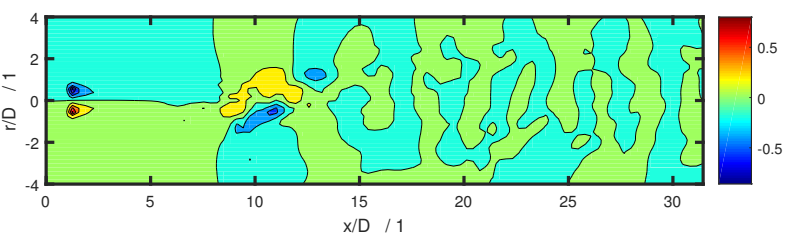

(d) xy plane, y velocity, imaginary part of DMD mode

Figure 4. Dynamic mode with $f=96 \mathrm{~Hz}$; y and $\mathrm{z}$ velocities

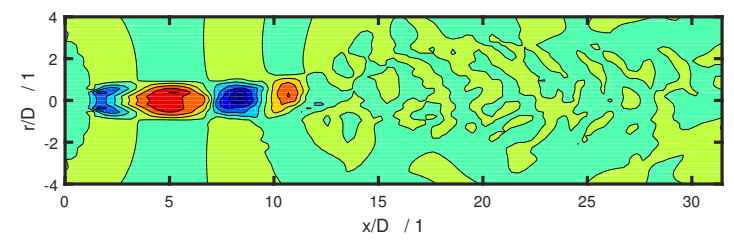

(a) xy plane, $x$ velocity, real part of DMD mode

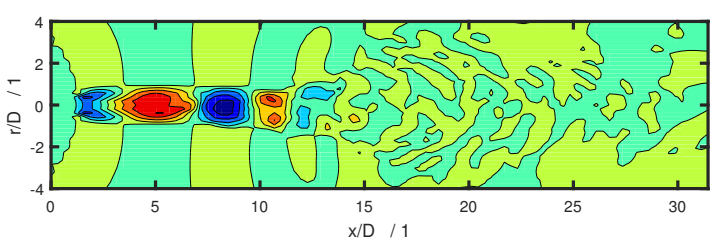

(c) $x z$ plane, $x$ velocity, real part of DMD mode
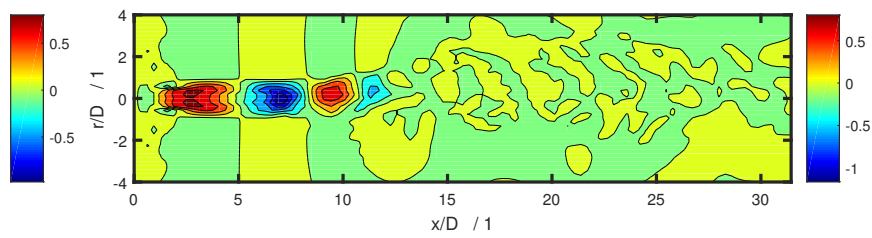

(b) xy plane, $x$ velocity, imaginary part of DMD mode
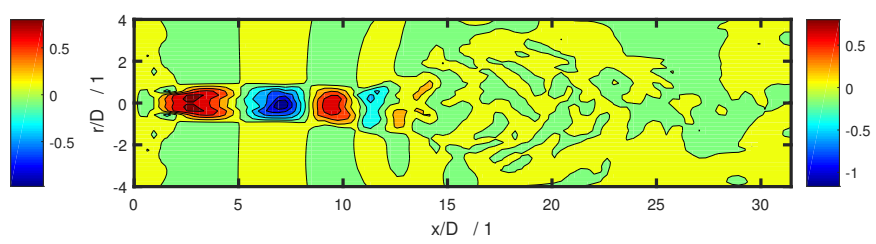

(d) $x z$ plane, $x$ velocity, imaginary part of DMD mode

Figure 5. Dynamic mode with $f=192 \mathrm{~Hz}$; axial velocity component $\mathrm{x}$ 


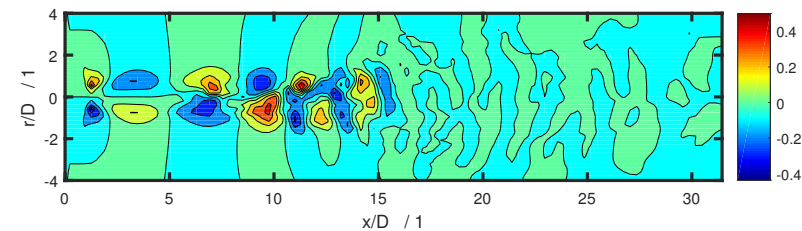

(a) xz plane, $z$ velocity, real part of DMD mode

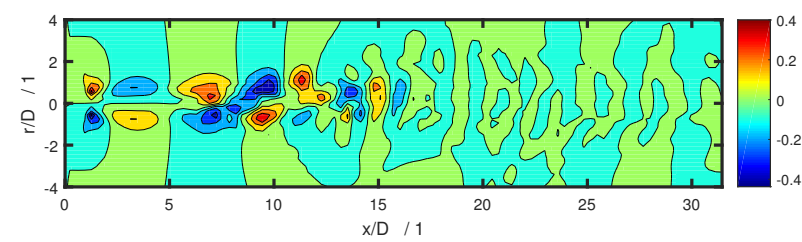

(c) xy plane, y velocity, real part of DMD mode

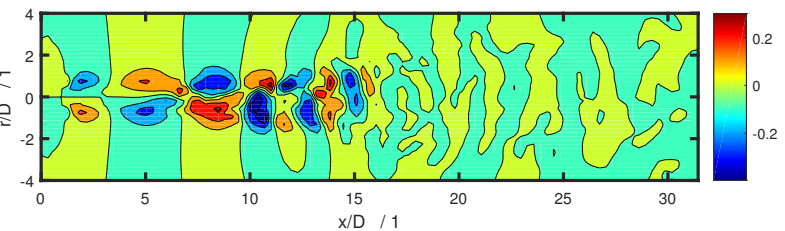

(b) xz plane, $\mathrm{z}$ velocity, imaginary part of DMD mode

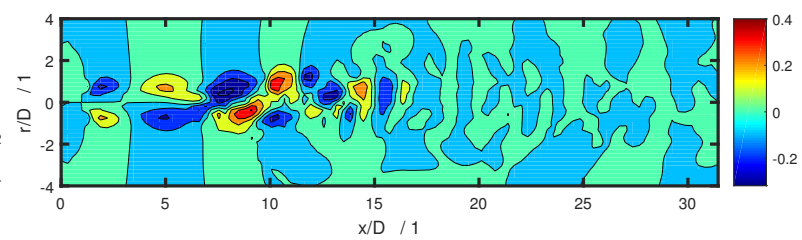

(d) xy plane, y velocity, imaginary part of DMD mode

Figure 6. Dynamic mode with $f=192 \mathrm{~Hz}$; y and z velocities

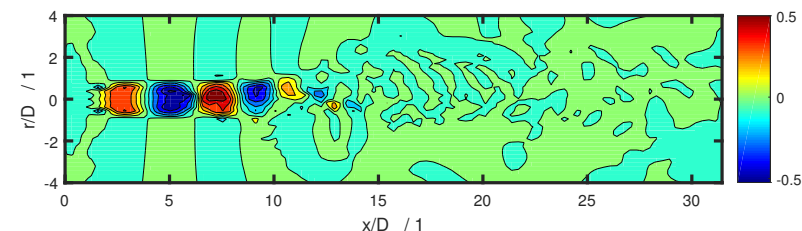

(a) xy plane, $x$ velocity, real part of DMD mode

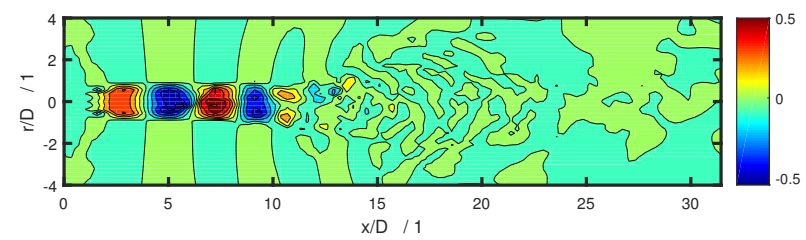

(c) xz plane, $x$ velocity, real part of DMD mode

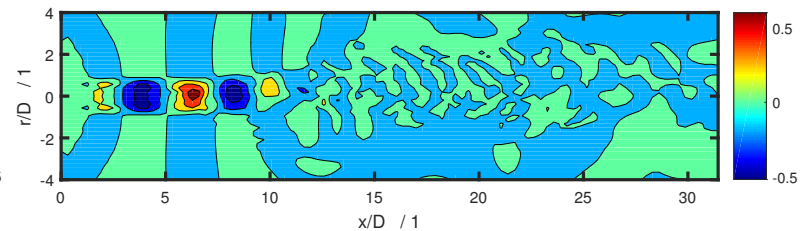

(b) xy plane, $\mathrm{x}$ velocity, imaginary part of DMD mode

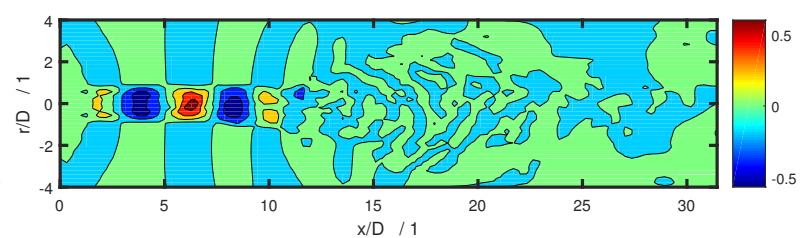

(d) xz plane, $x$ velocity, imaginary part of DMD mode

Figure 7. Dynamic mode with $f=288 \mathrm{~Hz}$; axial velocity component $\mathrm{x}$

\section{References}

[1] T. Hyhlík, P. Železný, J. Čížek, EPJ Web of Conferences, 45, (2013), 01043

[2] T. Hyhlík, H. Netřebská, EJP Web of Conferences, 114, (2016), 02045
[3] Z. Trávníček, Z. Broučková, J. Kordík, AIAA Journal, 50(9), (2012), 2012-2017.

[4] R. Holman, Y. Utturkar, R. Mittal, B. L. Smith, L. Cattafesta, AIAA Journal, 43(10), (2005), 2110-2116.

[5] P. J. Schmid, J. Fluid Mech., 656, (2010), 5-28. 